
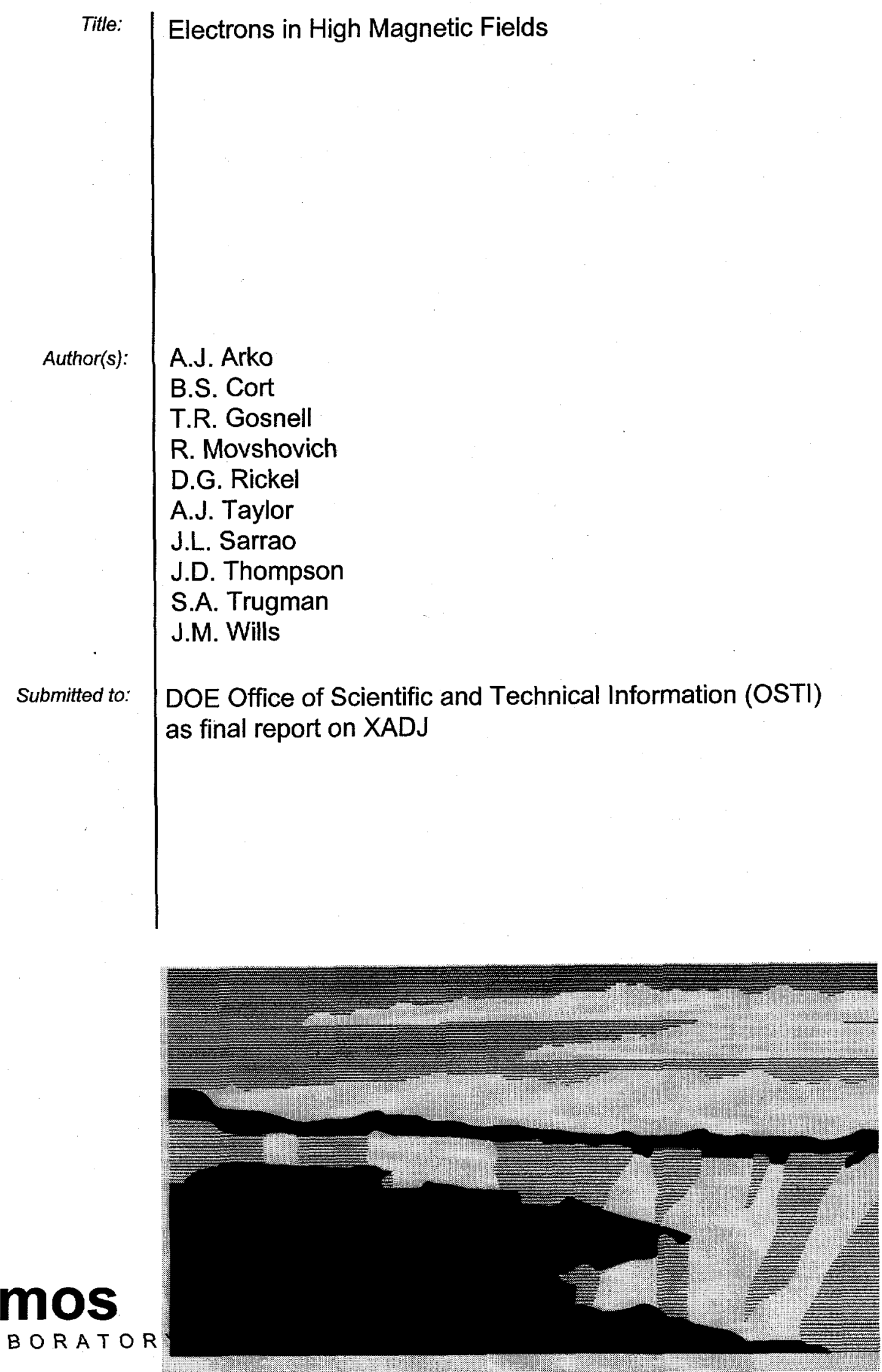

Los Alamos National Laboratory, an affirmative action/equal opportunity employer, is operated by the University of California for the U.S. Department of Energy under contract W-7405-ENG-36. By acceptance of this article, the publisher recognizes that the U.S. Government retains a nonexclusive, royalty-free icense to publish or reproduce the published form of this contribution, or to allow others to do so, for U.S. Government purposes. The Los Alamos National Laboratory requests that the publisher identify this article as work performed under the auspices of the U.S. Department of Energy. 


\section{DISCLAIMER}

This report was prepared as an account of work sponsored by an agency of the United States Government. Neither the United States Government nor any agency thereof, nor any of their employees, make any warranty, express or implied, or assumes any legal liability or responsibility for the accuracy, completeness, or usefulness of any information, apparatus, product, or process disclosed, or represents that its use would not infringe privately owned rights. Reference herein to any specific commercial product, process, or service by trade name, trademark, manufacturer, or otherwise does not necessarily constitute or imply its endorsement, recommendation, or favoring by the United States Government or any agency thereof. The views and opinions of authors expressed herein do not necessarily state or reflect those of the United States Government or any agency thereof. 


\section{DISCLAIMER}

Portions of this document may be illegible in electronic image products. Images are produced from the best available original document. 


\title{
Electrons in High Magnetic Fields
}

\section{RECEVED \\ $\mathrm{OCTO}_{4} 2020$ \\ OSTI}

\author{
AJ Arko (MST-10), BS Cort (NMT-5), TR Gosnell (MST-10), R Movshovich (MST-10), \\ DG Rickel (DX-6), AJ Taylor (MST-11), JL Sarrao (MST-10), \\ J.D Thompson (MST-10), S.A Trugman (T-11), and JM Wills (T-1)
}

\begin{abstract}
We performed experimental and theoretical work on the application of the National High Magnetic Field Laboratory (NHMFL) to the solution of fundamental unsolved problems in the many-body physics of condensed matter. Research was partitioned into two complementary but related projects: (1) The Electronic Structure of Actinide Intermetallics and (2) Femtosecond Dynamics of Many-Body Phenomena. With respect to (1), we focused on $4 \mathrm{f}$ and $5 \mathrm{f}$-based conducting materials that exhibit many-body correlations in their electronic properties. We measured the dHvA effect in 8 different materials, observing several magnetic transitions. Additionally we developed an apparatus capable of measuring the heat capacity of materials in the long pulse 60 tesla magnet. This gives us effective masses as a function of magnetic field. With respect to (2), the dynamic properties of electron correlations were studied using femtosecond laser techniques. In particular, we studied polaron formation and decay, as well as Cooper pair breaking and recombination.
\end{abstract}

\section{Backgroud and Resarch Objectives}

Materials in which coulomb and spin interactions are significant, display a host of exotic phenomena such as superconductivity, magnetism, metal-insulator transitions, anomalous equations of state, and heavy-fermion behavior. These phenomena-all attributable to dynamical correlations among conduction electrons in representative materials-currently comprise the frontier of modern condensed-matter theory and experiment; they represent the most fundamental of unsolved problems in the field. They illustrate how complex, scientifically rich, and technologically important effects can emerge when a large number of particles interact in a simple way.

This research focused on the application of the National High Magnetic Field Laboratory at Los Alamos to the solution of these fundamental problems. The goal was the development of measurement techniques via which it would be possible to delineate the electronic structure of $5 \mathrm{f}$ materials. Toward this end it was necessary to develop de Haas van Alphen probes capable of handling transuranic materials, heat capacity probes to 
measure heat capacity at high pulsed magnetic fields, and crystal growing apparatus for transuranics.

But high magnetic fields were not enough. We also applied — for the first timeultrafast optical techniques to the problem of correlated $f$ electrons in condensed matter; i.e., measurements of nonequilibrium properties such as transient reflectivity, transient transmissivity, and transient-induced cyclotron emission. The magnetic field was used as an independent control variable to tune the energy scale of the many-body interactions under study. Theoretical support for all these measurements was crucial. Explicitly stated, the scientific rationale for this research was the development of ultrafast optical methods in the study of $f$-electron and other many-body phenomena in high magnetic fields, motivated by outstanding scientific questions potentially resolvable with these techniques.

\section{Importance to LANL's Science and Technology Base and National R\&D Needs}

The primary long-term goal of this research was a determination of the electronic structure of the varous allotropes of $\mathrm{Pu}$ and its application to the equation of state of the material. Among the questions to be answered wre such as: Are the six structural phases of $\mathrm{Pu}$ a consequence or a correlate of electronic many-body effects? To what extent can electronic structure calculations be applied to understanding plutonium's equation of state? Does plutonium's notorious autocatalytic reaction with hydrogen have anything to do with the quantum-mechanical specifics of its $5 f$ electrons? What else about the chemistry of elemental plutonium is understandable in these terms? What insights into the metallurgical properties of the actinides become possible with first-principles understanding of their electronic structure and dynamics? Based on electronic properties can simple and inexpensive experiments be devised that will interrogate aging effects in nuclear materials?

The sequence of elements $\mathrm{U}, \mathrm{Np}, \mathrm{Pu}, \mathrm{Am}$ exhibits a transition from fully itinerant to fully localized behavior for $5 f$ electrons as the atomic number is increased. Plutonium lies at the boundary between these extremes; it therefore embodies the most intuitively elusive and theoretically intractable many-body physics of all the actinides. Motivated as much by the basic physics questions to be asked of $5 f$ electrons in actinide materials as by the opportunity to make singular scientific advances in support of the Laboratory's stewardship of the na- 
tional nuclear stockpile, the salient objective of this project was the measurement by dHvA of the $\delta$-plutonium Fermi surface. However, owing to their simpler handling and preparation, and to their proximity to $\mathrm{Pu}$ in the periodic table, requisite of this objective is the systematic investigation of elemental uranium and neptunium, followed by studies of intermetallic compounds formed of these elements in addition to those of plutonium, plus intermetallics formed with the $4 f$ correlated-electron element Ce.

Much of the information related to the correlated electronic structure of $\mathrm{Pu}$ was thus obtained via measurements on less radioactive systems, although it was not the hazardous nature of $\mathrm{Pu}$ that was the primary reason. While the apparatus for growing single crystals of $\mathrm{Pu}$ and its compounds was readily available, the necessary approvals and ES\&H concerns proved a much bigger obstacle. All approvals are at last available at the conclusion of this project, although, with the final availability o single crystals the project will continue in some form.

\section{Scientific Approach and Accomplishments}

\section{Experimental:}

a) dHvA Effect

We have built a rotating de Haas - van Alphen (dHvA) probe capable of accepting $\mathrm{Pu}$ samples. It has been thorughly tested using uranium heavy fermions from which we obtained a great deal of information regarding correlation effects in $5 \mathrm{f}$ systems. We observed dHvA oscillations in about 10 systems with some of the more interesting ones described here.

The largest effective mass measured $\left(15 \mathrm{~m}_{0}\right)$ was obtained in $\mathrm{USn}_{3}$, a single frequency which undoubtedly represents the lowest mass orbit in the system. Indeed, the $\mathrm{UX}_{3}(\mathrm{X}=\mathrm{Ga}$, $\mathrm{Al}, \mathrm{Sn}$ ) series of crystals produced illuminating results. It is undoubtedly the large variation in hybridization strength which manifests itself in the pronounced differences of these theree isostructural materials. While the $5 \mathrm{f}$ electrons in $\mathrm{UGa}_{3}$ are generally thought of as itinerant, in $\mathrm{UAl}_{3}$ and $\mathrm{USn}_{3}$ they are believed to undergo spin fluctuations. This is reflected both in the specific heat $\gamma$-values which increase across the series, and in the effective masses which subsequently regulate the amplitudes of the oscillations. The low-mass $\mathrm{UGa}_{3}$ system yields $10 \mathrm{dHvA}$ frequencies (see Figs. 1 and 2) as well as a meta-magnetic transition (Fig. 1), while 
the high-mass $\mathrm{UAl}_{3}$ and $\mathrm{USn}_{3}$ yield only a single frequency each, with no magnetic transitions. For $\mathrm{UGa}_{3}$ there is reasonable agreement with band calculations, although the magnetic transition is not predicted by the band model. Figure 1 shows raw data for $\mathrm{UGa}_{3}$ during a field pulse. The arrow points to the magnetic transition. Note that the data undergo a complete change above the transition. In Fig. 2 we show a Fourier transform of the data in Fig. 1. Note that there is a complete change in the frequency spectrum, indicating that the magnetic transition has completely altered the Fermi surface.

Other systems of interest are the $U_{C d} d_{11}$ heavy fermion system ( $\gamma$-value about $\left.800 \mathrm{~mJ} / \mathrm{Mole}-\mathrm{K}^{2}\right)$ as well as the CeRhIn5 ( $\gamma$-value about 200). The former represents the highest $\gamma$-value material for which the dHvA effect has been observed. Undoubtedly this is effected by the antiferromagnetic transition at $5 \mathrm{~K}$ which reduces the effective mass. Eleven frequencies were observed in this system as is shown in the Fourier transform of the data in Fig. 3. While this material likewise undergoes a magnetic transition, this time there is no change in the frequency spectrum, indicating no change in the poeriodicity. The transition thus may merely indicate a spin reorientation.

In the absence of single crystals we performed a magnetization measurement on $\delta$-Pu using the dHvA probe in a 50 Tesla pulse. No magnetic transitions were observed, but the exercise did strengthen the argument that $\mathrm{dHvA}$ in Pu-bearing specimens should pose no risk or unmanageable problem. We have considered the consequences of all this to the measurement of $\mathrm{Pu}$ samples. Our conclusion is that given a well-ordered single crystal, even with $\mathrm{Ga}$ impurities to stabiluze the delta phase, the observation of dHvA oscillations should pose no problem in field of about 50 Tesla.

b) Specific heat Measurements

Complimentary to the dHvA data we used specific heat probe for research in pulsed magnetic fields, developed earlier in this thrust, to study $\mathrm{Ce}_{3} \mathrm{Bi}_{4} \mathrm{Pt}_{3}$. This compound belongs to a class of Kondo Insulators, which are the $4 f$ and $5 f$ intermetallic compounds that present a relatively small temperature dependent gap in the electron excitation spectrum. This gap is a product of the interplay between electronic and magnetic correlations which can be suppressed by external magnetic fields. To test this, we measured the specific heat of $\mathrm{Ce}_{3} \mathrm{Bi}_{4} \mathrm{Pt}_{3}$ at temperatures down to $1.5 \mathrm{~K}$ and magnetic fields up to 60 Tesla. The 60TLP 
magnet, at the National High Magnetic Field Laboratory-Los Alamos National Laboratory, is driven by a $1.4 \mathrm{GW}$ synchronous power generator and produces a flat top field for a period of $100+$ ms at 60 Tesla and for longer time at lower fields. During the entire pulse the magnetic field varies at a maximum rate of $400 \mathrm{Tesla} / \mathrm{s}$ and its uniformity at the top field plateau is a fraction of a percent. We used a heat pulse method, where a known amount of heat is delivered to the calorimeter using the chip resistor. The result of a typical experiment on $\mathrm{Ce}_{3} \mathrm{Bi}_{4} \mathrm{Pt}_{3}$ is displayed in Fig. 4. The calorimeter comes to thermal equilibrium both before and after the heat pulse is delivered within the magnetic field plateau. The temperature is measured during the entire field pulse with a Cernox bare chip resistance thermometer. The heat capacity of the sample is determined as the ratio of the heat delivered to the sample to the change in its temperature due to the heat pulse. The results of the direct measurements of the specific heat in $\mathrm{Ce}_{3} \mathrm{Bi}_{4} \mathrm{Pt}_{3}$, accomplished in magnetic fields up to $60 \mathrm{Tesla}$, is displayed in Fig. 5. The zero field data agree well with earlier experiments. The observed increase in the heat capacity in fields equal to or larger than 40 Tesla is in very good agreement with the reversible temperature change due to the magnetocaloric effect observed during the pulse. Fig. 6 shows $\gamma_{\mathrm{H}}=\mathrm{C} / \mathrm{T}$ for $\mathrm{T} \rightarrow 0$, for $\mathrm{Ce}_{3} \mathrm{Bi}_{4} \mathrm{Pt}_{3}$ in magnetic fields up to 60 Tesla. A one parameter fit was done, fixing the lattice specific heat to its zero field value, which suggests a saturation of $\gamma$ at a value of $\gamma_{H}^{\text {sat }}=62 \pm 3 \mathrm{~mJ} /$ mole K$^{2}$. The strong threefold enhancement of $\gamma_{H}$ from its zero field value and the quantitative agreement with the estimate based on $T_{K}$ for a metallic ground state of $\mathrm{Ce}_{3} \mathrm{Bi}_{4} \mathrm{Pt}_{3}$ proves that we indeed crossed the Kondo insulatorKondo metal phase boundary.

This is the first report on direct specific heat measurements in pulsed magnetic fields, which has been accepted for publication in Nature. Development of the Specific Heat apparatus for use with the Long Pulse Magnet at the NHMFL significantly enhanced the range of capabilities available to the user community of this State-of-the-Art facility.

c) Crystal Growth

While the flux growth apparatus with which we plan to grow crystals of $\mathrm{Pu}$ compounds has been ready for almost tw years, the necessary approvals and ES\&H concerns required much more attention We are happy to report that final approvals are at last 
completed and crystal growth of $\mathrm{PuGa}_{3}$ and $\mathrm{Pu}_{3} \mathrm{Ga}$ will commence. The crystals will be used for a variety of other projects including photoemission, $\mathrm{dHvA}, \mathrm{NMR}$, resistivity magnetic susceptibility, surface studies, and heat capacity. All these projects are on-going under different funding agencies.

\section{d) Femtosecond Dynamics}

As part of this project we developed ultrafast optical techniques to determine the dynamics of quasiparticles in correlated electron materials in order to understand the underlying many body physics in these materials. To achieve this goal we carried out the following experiments. 1) We developed the technique of optical pump, terahertz probe spectroscopy to create a nonequilibrium distribution of quasiparticles in superconducting $\dot{\mathrm{Y}} \mathrm{Ba}_{2} \mathrm{Cu}_{3} \mathrm{O}_{7-\delta}(\mathrm{YBCO})$ and investigate the subsequent superfluid and quasiparticle dynamics. 2) We observed and characterizied terahertz emission from $\mathrm{YBa}_{2} \mathrm{Cu}_{3} \mathrm{O}_{7-\delta} \delta$ thin films excited by $1.5-\mathrm{eV}, 150$-fs pulses. 3) We carried out optical pump-probe measurements at $1.5 \mathrm{eV}$ and $3 \mathrm{eV}$ on the colossal magnetoresistance materials $\mathrm{La}_{0.7} \mathrm{Ca}_{0.3} \mathrm{MnO}_{3}$ and characterized the dynamics of laser-induced spectral weigth transfer in these materials. 4) We investigated the metal-insulator transition and the underlying spin dynamics through optical pump-probe techniques in $\mathrm{La}_{0.7} \mathrm{D}_{0.3} \mathrm{MnO}_{3}(\mathrm{D}=\mathrm{Ca}, \mathrm{Sr})$

In the first set of experiments we have developed the technique of optical pump, terahertz probe spectroscopy to create a nonequilibrium distribution of quasiparticles in superconducting $\mathrm{YBa}_{2} \mathrm{Cu}_{3} \mathrm{O}_{7-\delta}(\mathrm{YBCO})$ and investigate the subsequent superfluid and quasiparticle dynamics [P1-P4]. An initial fast recovery, occurring on a timescale of $10 \mathrm{ps,} \mathrm{is}$ followed a much longer process. The temperature dependence of the initial relaxation time is proportional to $1 / \Delta(T)$ for optimally doped $Y B C O$, but is constant below $T_{c}$ for underdoped YBCO. Further, by directly measuring conductivities, an evaluation of the transient temperature is determined after photoexcitation and after the initial recovery.

Previously, quasiparticle dynamics in YBCO have been studied using optical pump, optical probe spectroscopy. We create a nonequilibrium distribution of quasiparticles in superconducting $\mathrm{YBa}_{2} \mathrm{Cu}_{3} \mathrm{O}_{7-\delta}$ (YBCO) thin films using 150-fs, 1.5-eV optical pulses. The subsequent interplay between the superfluid and quasiparticle dynamics is investigated with coherent terahertz (THz) spectroscopy with the ultimate goal of understanding the character 
of the excited quasiparticles, their decay channels and relaxation times. Detection of the coherent $\mathrm{THz}$ probe enables the measurement of the evolution of the complex conductivity $\left(\sigma_{R}(\omega), \sigma_{I}(\omega)\right)$ over the frequency range from 0.2 to $0.8 \mathrm{THz}$ with picosecond resolution. The ensuing dynamics are interpreted using a two-fluid model where the conductivity below $T_{c}$ consists of a Drude-like component proportional to the number of quasiparticles and an imaginary component with $1 / \omega$ frequency dependence proportional to the superfluid population.

In Figure 7 we plot the initial recovery time, $\tau$, for $\mathrm{YBa}_{2} \mathrm{Cu}_{3} \mathrm{O}_{7}$ as a function of temperature for photoexcited densities of 2.5 and $5 \times 10^{19} \mathrm{~cm}^{-3}$. (To determine $\tau$ in a consistent way we fit the decay of the total $\mathrm{THz}$ field as a function of pump-probe delay to an exponential curve with decay time $\tau$ plus a constant.) Since the points for the two initial densities overlap, we conclude that this initial recovery process is relatively fluence independent. Moreover, the solid line in the Figure is proportional to $1 / \Delta(T)$ where $\Delta(T)$ is the superconducting order parameter calculated in the BCS weak coupling limit. This temperature dependence of the relaxation time has been predicted theoretically and observed in aluminum where the timescales are much longer. At $\mathrm{T}=4 \mathrm{~K}$ the recovery time of $1.85 \mathrm{ps}$ revealed in Figure 7 is limited by the 1 ps resolution of our experiment; a simple deconvolution yields a value of 1.5 ps. Measurements of the recovery time were also made on an underdoped sample of $\mathrm{YBa}_{2} \mathrm{Cu}_{3} \mathrm{O}_{6.5}$ where $\mathrm{T}_{\mathrm{c}}=55 \mathrm{~K}$. The recovery time for $\mathrm{YBa}_{2} \mathrm{Cu}_{3} \mathrm{O}_{6.5}$, also shown in Figure 7, is temperature independent and about a factor of two larger than observed for the optimally doped material, consistent with the presence of a pseudogà in underdoped cuprates.

In a second set of measurements, we report the first observation of terahertz emission from $\mathrm{YBa}_{2} \mathrm{Cu}_{3} \mathrm{O}_{7-\delta}$ thin films excited by $1.5-\mathrm{eV}, 150$-fs pulses in the absence of external electric or magnetic fields [P5, P6]. The terahertz waveforms observed from these films when excited by $150 \mathrm{fs}, 1.5 \mathrm{eV}$ pulses are always bipolar with a width limited by the 1-ps resolution of the photoconducting detector. Th emitted terahertz electric field scales linearly with incident intensity up to fluences of $4 \mathrm{~mJ} / \mathrm{cm}^{2}$, consistent with a $\chi^{2}$ process; however since YBCO is centrosymmetric, the bulk electric dipole contribution to $\chi^{2}$ vanishes. Since different exhibit distinctive dependences on incident and detected polarizations, as well as on 
orientation of crystal axes, we measure peak-to-peak emitted terahertz field as a function of incident and detected polarizations about the surface normal, $\phi$. The result for $\mathrm{YBa}_{2} \mathrm{Cu}_{3} \mathrm{O}_{7}$ at $300 \mathrm{~K}$ is shown in Figure 8 . The strong anisotropy with $4 \phi$ symmetry $(\cos (4 \phi)$ for Pdetected emission and $\sin (4 \phi)$ for S-detected emission) in the emitted field demonstrates that this emission is generated by optical rectification due to the bulk electric quadrupole and magnetic dipole source terms, comprising the first observation of such source terms in terahertz emission

This emission is characterized for optimally doped $\delta=0$, and underdoped $\delta=0.5$ films, over a range of temperatures $\mathrm{T}, 4 \mathrm{~K}<\mathrm{T}<300 \mathrm{~K}$ where the films change from superconductors to correlated metals. The temperature dependence of the $\mathrm{THz}$ emission for optimally doped films is consistent with a two-fluid model where the ratio of the superfluid to normal emission efficiency is 1.65. The temperature dependence of the emission from the underdoped sample is not described by a simple two-fluid model, but is consistent with a pseudogap opening at $150 \mathrm{~K}$ in the underdoped material.

A model of this emission assuming that the nonlinearity results from transport of carriers with different masses and relaxation times in the three crystallographic directions is consistent with the data. A fit of the data to this model yields values for the anisotropic masses and relaxation times in $\mathrm{YBa}_{2} \mathrm{Cu}_{3} \mathrm{O}_{7-\delta} \delta$ thin films. A significantly higher value for the c-axis conductivity is found, $\sigma_{c}=0.152 \mathrm{eV}$ for $\mathrm{YBa}_{2} \mathrm{Cu}_{3} \mathrm{O}_{7}$ and $\sigma_{\mathrm{c}}=0.159 \mathrm{eV}$ for $\mathrm{YBa}_{2} \mathrm{Cu}_{3} \mathrm{O}_{6.5}$, as compared to the literature value [1] of $\sigma_{\mathrm{c}}=0.046 \mathrm{eV}$. A higher value for the ratio of the chain to plane conductivities $\sigma_{b} / \sigma_{a}$ results from this data with a ratio of 2.7 for $\mathrm{YBa}_{2} \mathrm{Cu}_{3} \mathrm{O}_{7}$ and 3.3 for $\mathrm{YBa}_{2} \mathrm{Cu}_{3} \mathrm{O}_{6.5}$, as compared to 2.2 for $\mathrm{YBa}_{2} \mathrm{Cu}_{3} \mathrm{O}_{7}$ from Ref. 1 . This apparent discrepancy may be caused by the difference in conductivities between untwinned single crystals and twinned thin films. We further note that the measurement of anisotropic conductivity in twinned samples is problematic using linear transport measurements.

In a third set of experiments, we carried out optical pump-probe measurements at 1.5 $\mathrm{eV}$ and $3 \mathrm{eV}$ on the colossal magnetoresistance materials $\mathrm{La}_{0.7} \mathrm{Ca}_{0.3} \mathrm{MnO}_{3}$. The material response is determined in the femtosecond to the nanosecond temporal regimes. By analyzing the absorption and refractive dynamics within the Kramers-Kronig transformations 
we demonstrated dynamic spectral weight transfer from the intraband Drude component to the interband transitions. This spectral transfer and its strongly temperature dependent dynamics are discussed in the framework of photoinduced demagnetization due to the exchange of energy and thermal equilibration among the electronic, lattice and spin systems [P7]. This effect is observed in other manganites with different transition temperatures. The measured dynamics are found to be insensitive to the excitation photon energy.

The measured response probed at $3 \mathrm{eV}$ (outside the range of spectral weight transfer (SWT) accompanying the ferromagnetic transition) reveals induced absorption with similar dynamics in the paramagnetic insulating and the ferromagnetic state. On the other hand, the measured response at $1.5 \mathrm{eV}$ (within the range of the SWT) exhibits a strong sensitivity to the metal insulator transition. A negative long-lived transient (absorption bleaching) is observed in the PI state while for $T \leq T_{\mathrm{c}}$ a positive transient (increased absorption) develops below $T_{\mathrm{c}}$. This component is observed only in the FM state were spectral weight has transferred into the low energy Drude contribution. Using Kramers-Kronig arguments we argue that this component originates from the onset of partial reversal of the SWT from the Drude region to the $1.5 \mathrm{eV}$ probe region upon perturbing the sample. We call this reversal dynamic spectral weight transfer (DSWT). In this context the increase of resistance upon photoexcitation in the ferromagnetic state, indicates a suppression of the Drude spectral weight and is consistent with DSWT.

Finally, the metal-insulator transition and the underlying spin dynamics were investigated through optical pump-probe technique in $\mathrm{La}_{0.7} \mathrm{D}_{0.3} \mathrm{MnO}_{3}(\mathrm{D}=\mathrm{Ca}, \mathrm{Sr})[\mathrm{P} 8$ ]. Measurements are done at temperatures spanning the phase transition and in magnetic fields up to 7 Tesla. The dynamics of the optically induced spectral weight transfer is shown to follow the temperature dependence of the spin specific heat with no significant temperature dependence of the spin-lattice coupling. The increase of itinerancy accompanying the development of the ferromagnetic metallic state is also evident.

The dynamics observed in our measurements has two components; an ultrafast one and a slower component with a strongly temperature dependent time constant. We attribute the ultrafast component, $<1 \mathrm{ps,}$ to the electron-spin demagnetization whereas the slower component, $\sim 20-200 \mathrm{ps}$, is related to the spin-lattice thermalization process. This association of DSWT with the spin dynamics is justified in these materials by experiments that show 
strong correlation of magnetization with resistivity and thermal conductivity. It is also consistent with the prevailing CMR theories that attribute the metal-insulator transition to either the magnetization induced increase in the electron conduction bandwidth, the magnetization induced current carrier density collapse (CCDC) through the breakup of immobile singlet bipolarons, or a magnetization dependent localization length or barrier. These different theories have demonstrated their ability to capture the essence of the experimentally observed CMR effect and SWT as a function of temperature and magnetic field.

The spin-lattice thermalization time constant (i.e. slow component) is plotted in fig. 9 for the $\mathrm{La}_{0.7} \mathrm{Ca}_{0.3} \mathrm{MnO}_{3}$ (LCMO) and the $\mathrm{La}_{0.7} \mathrm{Sr}_{0.3} \mathrm{MnO}_{3}$ (LSMO) film as a function of the temperature normalized to their respective $T_{c}$. The time constant in LCMO peaks at $\sim 260 \mathrm{~K}$ with a value of $\sim 280$ ps that decreases to $\sim 20$ ps at low temperatures. The extracted time constant for LSMO also peaks below $T_{c}$.

\section{Theory:}

a) Band Structure calculations

We have performed a number of band structure calculations in support of the $\mathrm{dHvA}$ effort. Among these are UGa3 and CeRhIn5 which indicated reasonably good agreement between theory and experiment. The latter material is an unusual heavy fermion system in that it undergoes an antiferromagnetic transition at ambient pressure, but becomes superconducting under pressure. The good agreement with the dHvA frequencies suggests that the bans structure is corret which then implies that there are large regions in momentum space where there are no bands of $5 \mathrm{f}$ character. This is highly unusual for a heavy fermion with a $\gamma$-value of 200 , and implies that there is much as yet not understood regarding heavy fermion behavior. The work UGa3 was done in collaboration with Peter Oppeneer of Dresden.

We have performed detailed band calculations for $\alpha$ - and $\delta$-Pu but there are no dHvA data with which to compare the results. Instead in Fig. 10 we compare the density of states to a photoemission spectrum. The calculated data have been appropriately broadened for a comparison to the data. The agreement appears reasonable, although much work remains to be done 


\section{b) Femtosecond Dynamics}

The theory was largely focused on the second area of the two-fold DR approach: Femtosecond dynamics of electron-electron correlations, i.e. many-body phenomena, in high magnetic fields.Part of the theoretical effort was very closely coupled to the DR experiments on ultrafast optical and terahertz probes of correlated electron systems (see papers coauthored with Taylor et al. in publication list). We explained the nonlinear generation of terahertz radiation in high temperature superconductors, including the angle, polarization, and doping dependence. The measurements provide information on the nonlinear, anisotropic dynamics of free electrons in the locally orthorhombic, twinned films, and their emission of electric quadrupole and magnetic dipole radiation. The calculation treats the double refraction of the incoming beam, and the absorption and nonlinear interaction of the resulting components. Other work measured and interpreted the fast dynamics of the destruction and reformation of Cooper pairs in YBCO thin films by an intense optical pulse. Another part of the theoretical effort explored the fast quantum dynamics of the many-body system resulting when electrons are coupled to phonons. We calculated the ground and excited states of the resulting polaron quasiparticle, including a phase transition in the first excited state where the polaron absorbs an additional phonon. We invented a novel algorithm that calculates the ground state energy more quickly and 10,000,000 times more accurately than previous work. We also calculated how a polaron quasiparticle emit phonons and falls apart when driven far from equilibrium. By integrating the many-body time-dependent Schrodinger equation, we calculated in real space and time how a bare electron (created for example by an optical pulse) emits phonons and becomes a polaron. We also calculated when and how two polarons bind together by exchanging dynamical, quantum phonons in the presence of Coulomb repulsion. 


\section{Publications}

1. Zang, J., H. Rhoder, A. R. Bishop, and S. A. Trugman, "Low Temperature Magnetic Properties of the Double Exchange Model," J. Phys.: Cond. Matt. 9, L157 (1997).

2. Bonča, J. and S. A. Trugman, "Inelastic Quantum Transport," Phys. Rev. Lett. 79, 4874 (1997).

3. Balatsky, A.V. and S. A. Trugman, "Lifshitz tail in the density of states of a superconductor with magnetic impurities," Phys. Rev. Lett. 79, 3767 (1997). \% LAUR-97-1749

4. Zang, J., S. A. Trugman, A. R. Bishop, H. Roder, "Persistent Current in the Ferromagnetic Kondo Lattice Model," Phys. Rev. B 56, 11839 (1997). \% LAUR-97-2224

5. Darling, T.W., A. Migliori, E. G. Moshopoulou, S. A. Trugman, J. J. Neumeier, J. L. Sarrao, A. R. Bishop, and J. D. Thompson, "Measurement of the elastic tensor of a single crystal of $\mathrm{La}_{0.83} \mathrm{Sr}_{0.17} \mathrm{MnO}_{3}$ and its response to magnetic fields", Phys. Rev. B 57, 5093 (1998). \% LAUR-97-1912

6. Siders, J.L.W., S. A. Trugman, R. J. Houlton, F. H. Garzon, and A. J. Taylor, "'Terahertz Emission from $\mathrm{YBa}_{2} \mathrm{Cu}_{3} \mathrm{O}_{7-\delta}$ Thin Films via Bulk Electric Quadrupole Optical Rectification", Technical Digest Summaries of papers presented at the International Quantum Electronics Conference, Technical Digest Series, Vol. 7, p. 149, May 1998, Optical Society of America. \% LAUR-97-4680

7. Trugman, S.A. and Janez Bonča, "The Polaron: Ground State, Excited States, and Far From Equilibrium", J. Superconductivity 12, 221 (1999). \% LAUR-98-2572

8. Bonča, J., S. A. Trugman, and I. Batistic, 'Holstein Polaron", Phys. Rev. B 60, 1633 (1999). $\%$ LA-UR-98-5640

9. Mustre de Leon, J., R. DeCross, A. Rubio-Ponce, A. R. Bishop, S. A. Trugman, 'Isotopic Shifts in a Polaronic System", J. Superconductivity 12, 37 (1999). \% LAUR-98-3803

10. Mustre de Leon, J., R. de Coss, A. R. Bishop, and S. A. Trugman, "Isotopic Substitution in a Model Polaronic System", Phys. Rey. B 59, 8359 (1999). \%LA-UR-98-5639

11. Siders, J.L., R. N. Jacobs, C. W. Siders, S. A. Trugman, and A. J. Taylor, "Nonequilibrium Superconductivity and Quasiparticle Dynamics in $\mathrm{YBa}_{2} \mathrm{Cu}_{3} \mathrm{O}_{7-\delta}$ " Ultrafast Phenomena XI (Springer-Verlag, Berlin, 1998), p. 365.

12. Modler, R., J. D. Thompson, S. A. Trugman, D. G. Rickel, P. C. Canfield, and Z.

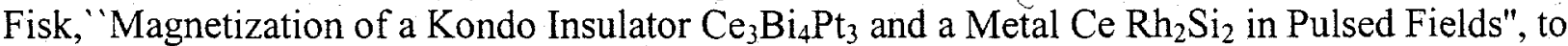
appear in Proceedings of the International Conference on Physical Phenomena at High Magnetic Fields, Tallahassee, Florida, Oct. 1998. 
13. Bonča, J., T. Katrasnik, and S. A. Trugman, "Mobile Bipolaron", submitted to PRL.

14. Jaime, M., R. Movshovich, G. R. Stewart, M. Gomez-Berisso, W. P. Beyermann, P. C. Canfield, and J. L. Sarrao, "Direct Specific Heat Measurements up to 60 Tesla in $\mathrm{Ce}_{3} \mathrm{Bi}_{4} \mathrm{Pt}_{3}$ Kondo Insulator", accepted by Nature.

15. Jaime, M., R. Movshovich, J. L. Sarrao, G. Stewart, J. Kim, and P. C. Canfield, "Direct Heat Capacity Measurement in up to 60 Tesla in $\mathrm{Ce}_{3} \mathrm{Bi}_{4} \mathrm{Pt}_{3}$ ", Physica B, in press.

16. Cornelius, A.L., A.J. Arko, J.L. Sarrao, N Harrison, "de Haas-van Alphen effect, magnetic transitions and specific heat in the heavy fermion system $U_{C d}$ ", Phys. Rev. B 59, 13542 (1999).

17. Cornelius, A.L., A.J. Arko, J.L. Sarrao, N Harrison, "de Haas-van Alphen effect, magnetic transitions and specific heat in the heavy fermion system UCd 1 ", Phys. Rev. B 59, 13542 (1999).

18. Cornelius, A.L., A.J. Arko, J.L. Sarrao, M.F. Hundley, "de Haas van Alphen effect, magnetic transitions and specific heat in the quasi-two dimensional heavy fermion antiferromagnet CeRhIn5", Submitted to PRB.

19. Siders, C.W., J. L. W. Siders, A. J. Taylor, S.-G. Park, and A. M. Weiner, "Efficient highenergy pulse train generation using a $2^{\mathrm{n}}-$ Michelson interferometer," Appl. Opt. 37, 5302 (1998).

20. Park, S.-G., A. M. Weiner, M. R. Melloch, C. W. Siders, J.L. W. Siders, and A. J. Taylor "High-power narrow-band terahertz generation using large-aperture photoconductors, IEEE J. Quantum Electron. 35, 1257-1268 (1999).

21. Lobad, A.L., A. J. Taylor, and C. Kwon, "Ultrafast spin dynamics in colossal magnetoresistance manganites," submitted to Phys. Rev. Lett. (2000).

22. Averitt, R.D., G. Rodriguez, J. L. Siders, S. A. Trugman, and A. J. Taylor, "Conductivity artifacts in optical-pump $\mathrm{THz}$ probe measurements in $\mathrm{YBa}_{2} \mathrm{Cu}_{3} \mathrm{O}_{7}$," J. Opt. Soc. Am. B 17, 327$331(2000)$.

23. Siders, J.L., T. R. Gosnell, S. A. Trugman, and A. J. Taylor, "Terahertz Emission from $\mathrm{YBa}_{2} \mathrm{Cu}_{3} \mathrm{O}_{7-\delta}$ ", Trends in Optics and Photonics 13, Ultrafast Electronics and Optoelectronics, Martin Nuss and John Bowers, eds., (Optical Society of America, Washington, DC, 1997) pp. $252-256$.

24. Siders, J.L., S.A. Trugman, F.H. Garzon, R. J. Houlton, and A. J. Taylor, "Terahertz emission from $\mathrm{YBa}_{2} \mathrm{Cu}_{3} \mathrm{O}_{7} \delta$ thin films via bulk electric quadrupole/magnetic dipole optical rectification," submitted to Phys. Rev. B (1999). 
25. Lobad, A.I., A. J. Taylor, C. Kwon, S. A. Trugman, and T. R. Gosnell, "Laser-induced dynamic spectral weight transfer in $\mathrm{La}_{0.7} \mathrm{Ca}_{0.3} \mathrm{MnO}_{3}$," Chem. Phys. 251, 227-236 (2000). Invited Paper.

26. Siders, J.L.W., R. N. Jacobs, C. W. Siders, S. A. Trugman, and A. J. Taylor, "Nonequilibrium superconductivity and quasiparticle dynamics in $\mathrm{YBa}_{2} \mathrm{Cu}_{3} \mathrm{O}_{7-} \delta$," in Ultrafast Phenomena XI, T. Elsaesser, J. G. Fujimoto, D. A. Wiersma, and W. Zinth, eds. (Springer, New York, 1998) pp. 365-367.

27. Jaime, M., et al, "Heat capacity measurements in pulsed magnetic fields", Proceedings of the PPHMF-III Conference, Eds. Z. Fisk, L. Gor'kov and R. Schrieffer p. 148, 1999.

28. Lobad, A.I., et al., "On the Coherent Phonon Generation Mechanism in $\mathrm{YBa}(2) \mathrm{Cu}(3) \mathrm{O}(7)$ " (submitted to Phys. Rev. B).

\section{Figure Captions}

Fig. 1 trace of pickup coil output for a (100) direction of UGa3. The y-axis is the voltage induced in the pickup coil during the magnetic field pulse, with the oscillations being a consequence of the oscillatory magnetization. The $\mathrm{x}$-axis is the inverse of the magnetic field. The arrow points to the position in inverse field of the magnetic transition chracterized by the discontinuous change in the slope of the magnetization. Note that there is a complete change in the character of the oscillations above and below the transition, meaning that the Fermi surface has been completely modified.

Fig.2 Fourier transform of the pulse in figure 1 above and below the magnetic transition at $\mathrm{B}_{\mathrm{M}}$. This emphasizes the complete change in the frequency spectrum above and below $\mathrm{B}_{\mathrm{M}}$.

Fig. 3 Fourier transform of the pickup coil output for a (100) direction of UCd11There are two magnetic transitions in this material $\left(\mathrm{B}_{\mathrm{M} 1}\right.$ and $\left.\mathrm{B}_{\mathrm{M} 2}\right)$ but oscillations are only observed above $B_{M 2}$ where $B$ is of sufficient strength to yield the dHvA effect. The frequency spectrum, though much weaker below $B_{M 2}$ due to the lower magnetic field, is essentially unchanged despite the magnetic transition. It is expected that only a spin reorientation occurs, with no change in periodicity.

Fig. 4 Heat capacity experiment in the 60TLP magnet. The magnetic field profile is displayed in red, the temperature of the $\mathrm{Ce}_{3} \mathrm{Bi}_{4} \mathrm{Pt}_{3}$ (sample \#1) in green, and eight (10 ms each) heat pulses delivered during the field flat top in blue. The temperature decreases during the field ramp due to the adiabatic magnetization of the sample, reaches equilibrium before and after each heat pulse is delivered, and increases during the field ramp down due to the adiabatic demagnetization of the sample. 
Fig. 5 Specific heat divided by the temperature vs $\mathrm{T}^{2}$ for $\mathrm{Ce}_{3} \mathrm{Bi}_{4} \mathrm{Pt}_{3}$ single crystal sample \#2 in magnetic fields between zero and 60 Tesla after subtraction of small addenda. Dashed lines are guides to the eye.

Fig. 6 Sommerfeld coefficient extracted from the data displayed in Fig. 3. Solid symbols (a) correspond to a two parameters fit described in the text. Open symbols ( ) were obtained with a single parameter fit.

Fig. 7 Initial recovery time, $\tau$, for $\mathrm{YBa}_{2} \mathrm{Cu}_{3} \mathrm{O}_{7-\delta}$ where $\delta=0$ and 0.5 a function of temperature.

Fig. 8 Peak-to-peak $\mathrm{THz}$ field amplitude versus azimuthal angle, $\phi$, for a $\mathrm{YBa}_{2} \mathrm{Cu}_{3} \mathrm{O}_{7}$ film excited by P- (filled symbols) or S-polarized (open symbols), $1.5-\mathrm{eV}$ pulses at an incident angle of $50^{\circ}$ and detecting P- (circles) or S-polarized (rectangles) radiation. The solid curves are fits to the data using the theoretical model.

Fi. 9 Spin-lattice relaxation constants for LCMO (square) and LSMO (circle) as a function of the temperature normalized to Tc. The magnetic specific heat (dashed lines) is superimosed on $\tau_{\mathrm{sL}}$ for both LCMO and LSMO.

Fig. 10 Comparison of the calculated density of states (DOS) of $\alpha$ - and $\delta$-Pu to photoemission spectra in the absence of quantum oscillations (single crystals not yet available). The calculated DOS has been appropriately smoothed to approximate instrument resolution as well as inherrent broadenings associated with photoemission. Though the $\delta$-Pu calculation is based on an ad hoc model in which four of he five $5 \mathrm{f}$ electrons are constrained to be localized, surprisingly the results appear to capture the essential features of the spectrum. By contrast, the $\alpha$-Pu calculation in which all the $5 \mathrm{f}$-electrons are allowed to be itinerant, as anticipated from bulk property measurements, appears to miss the narrow feature at the Fermi energy.

Fig. 11 Frames from a movie showing the many-body quantum dynamics of polaron formation after a free electron is injected (for example by optical excitation in manganites). At the time of the middle frame, there is a substantial amplitude for both a free electron state and a polaron state, as can be seen in the double bump structure. The polaron amplitude increases with time. The calculation is done by integrating the time-dependent many-body Schrodinger equation with local (Holstein) electron-phonon coupling and periodic boundary conditions on 30 sites, shown in a doubled system of 60 sites. 


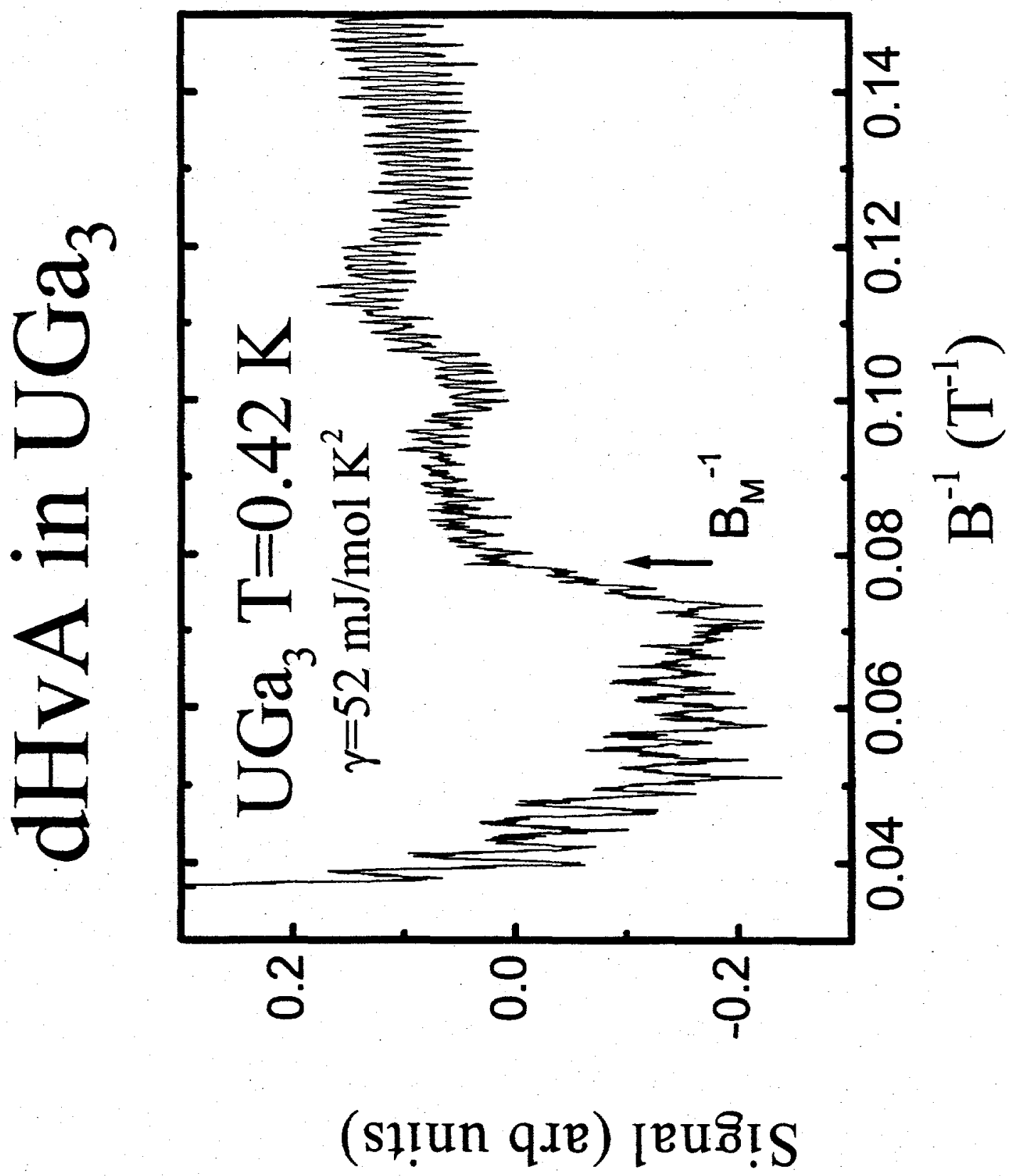




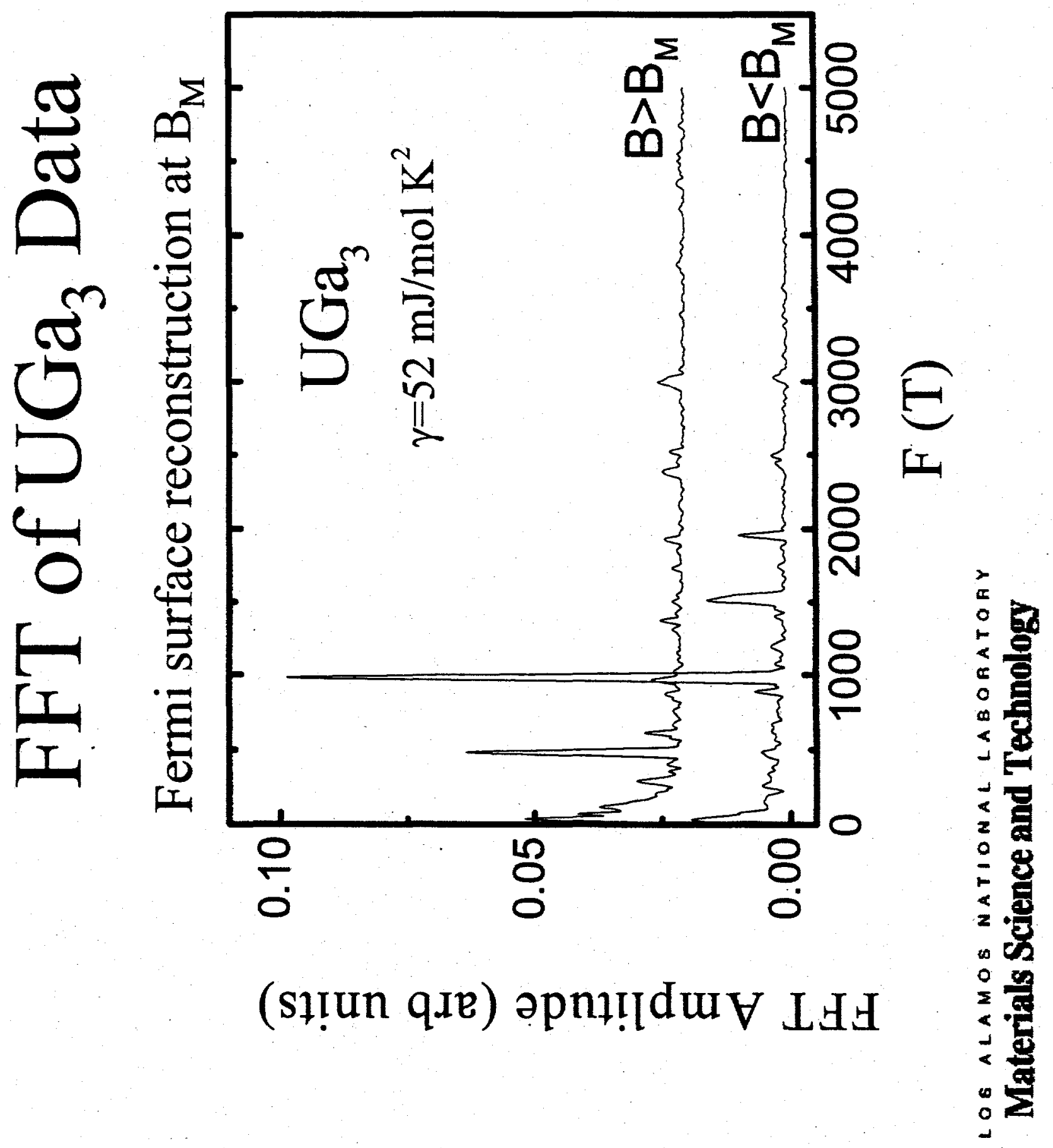




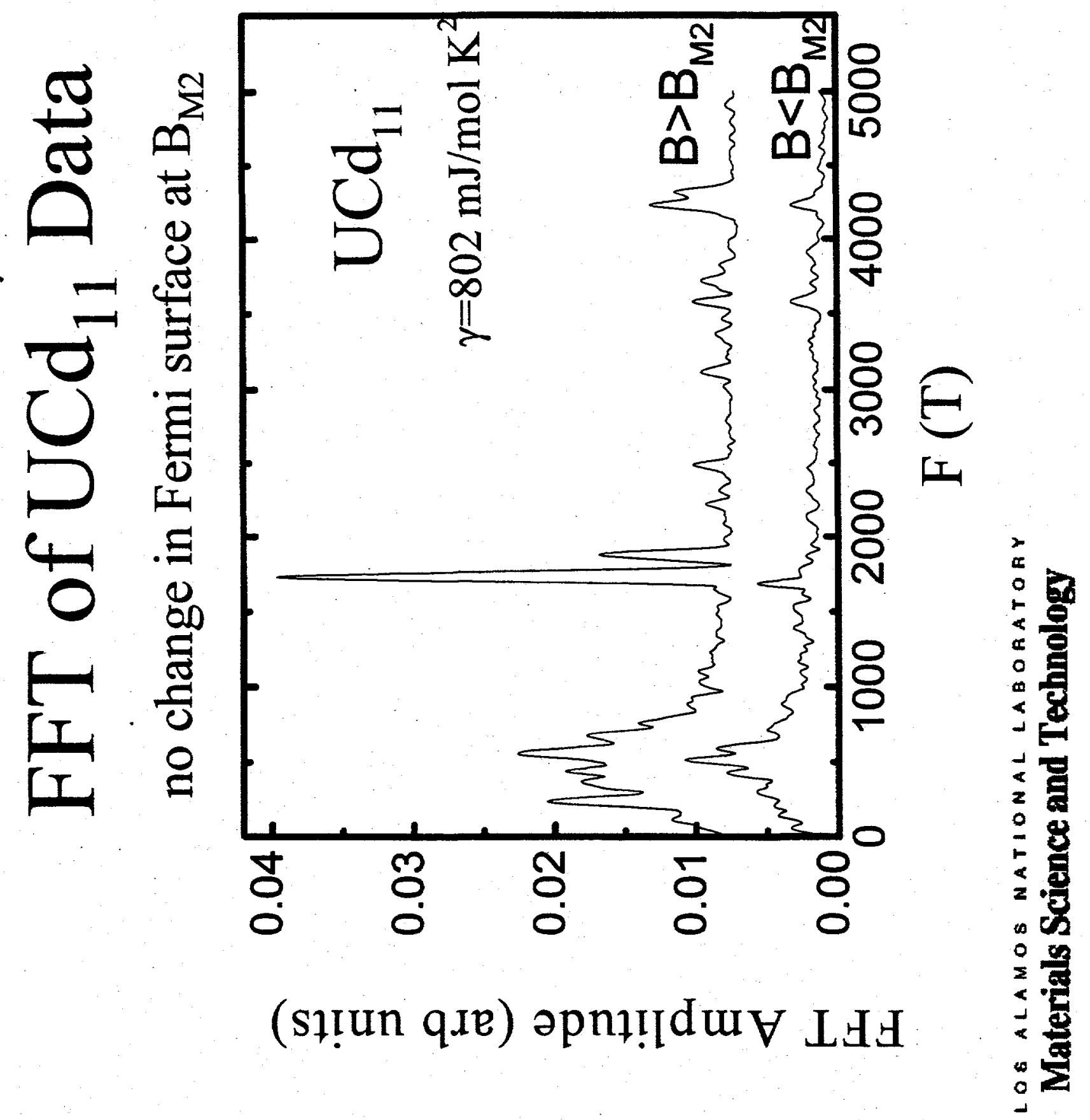


Heater Voltage (V)
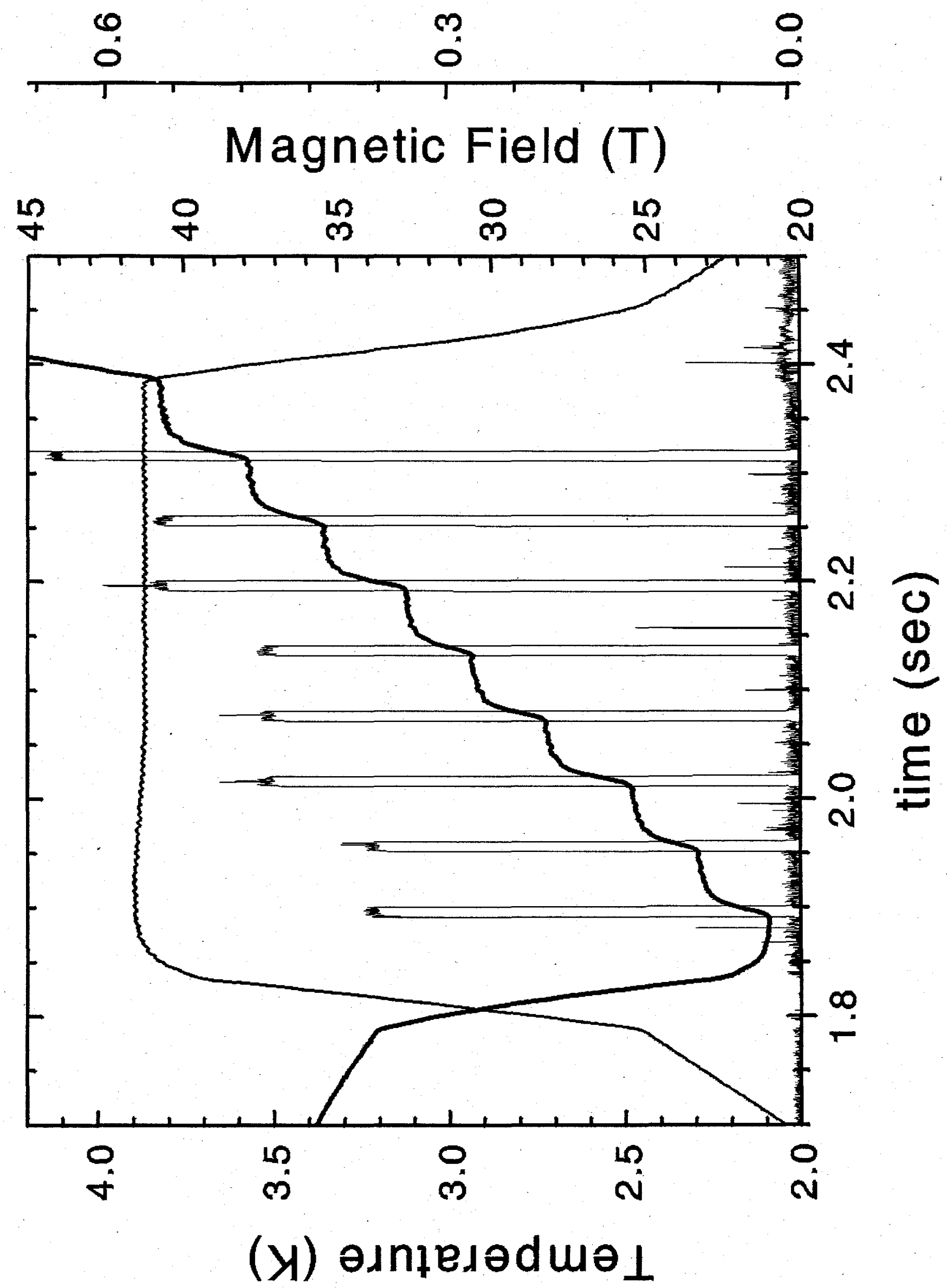


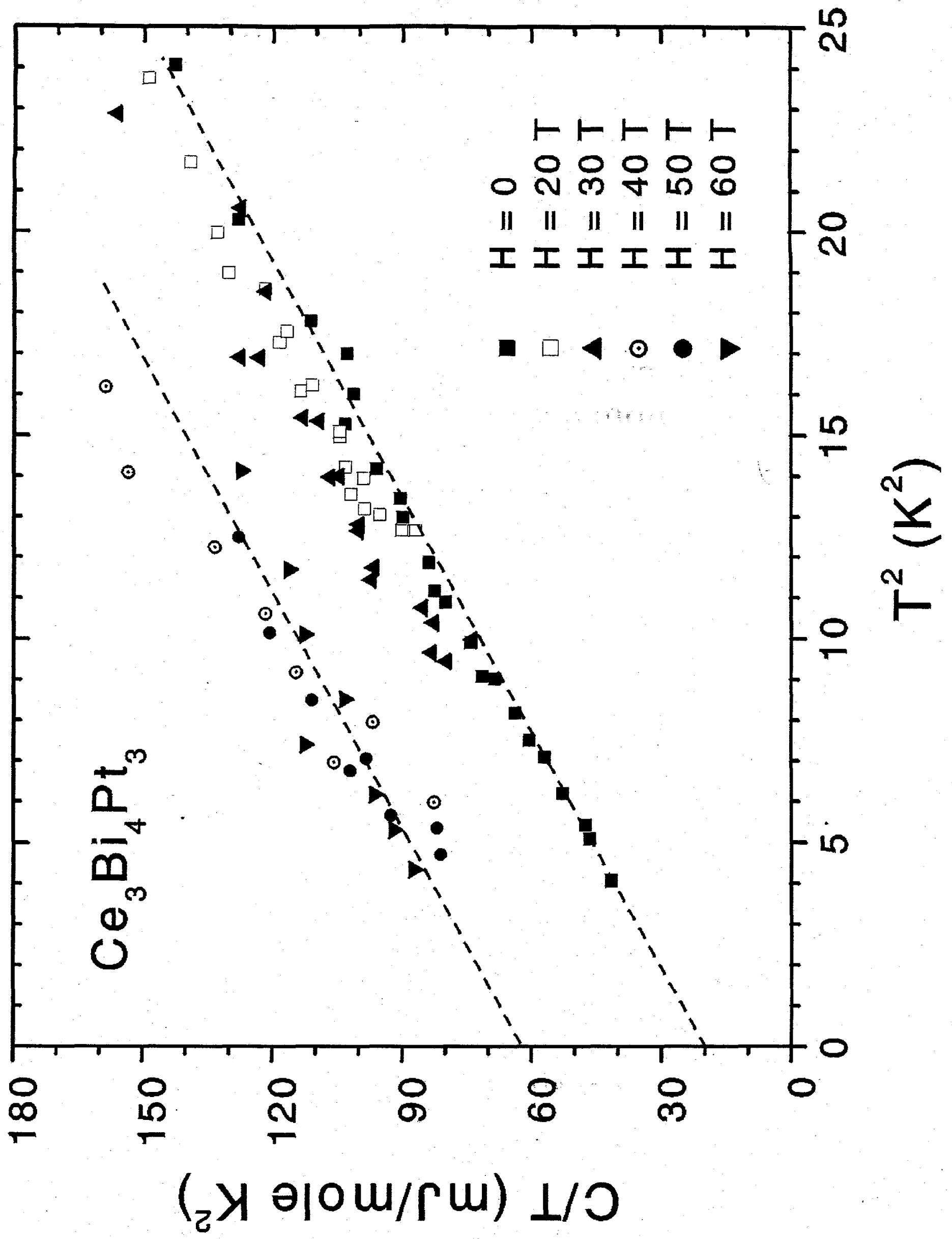




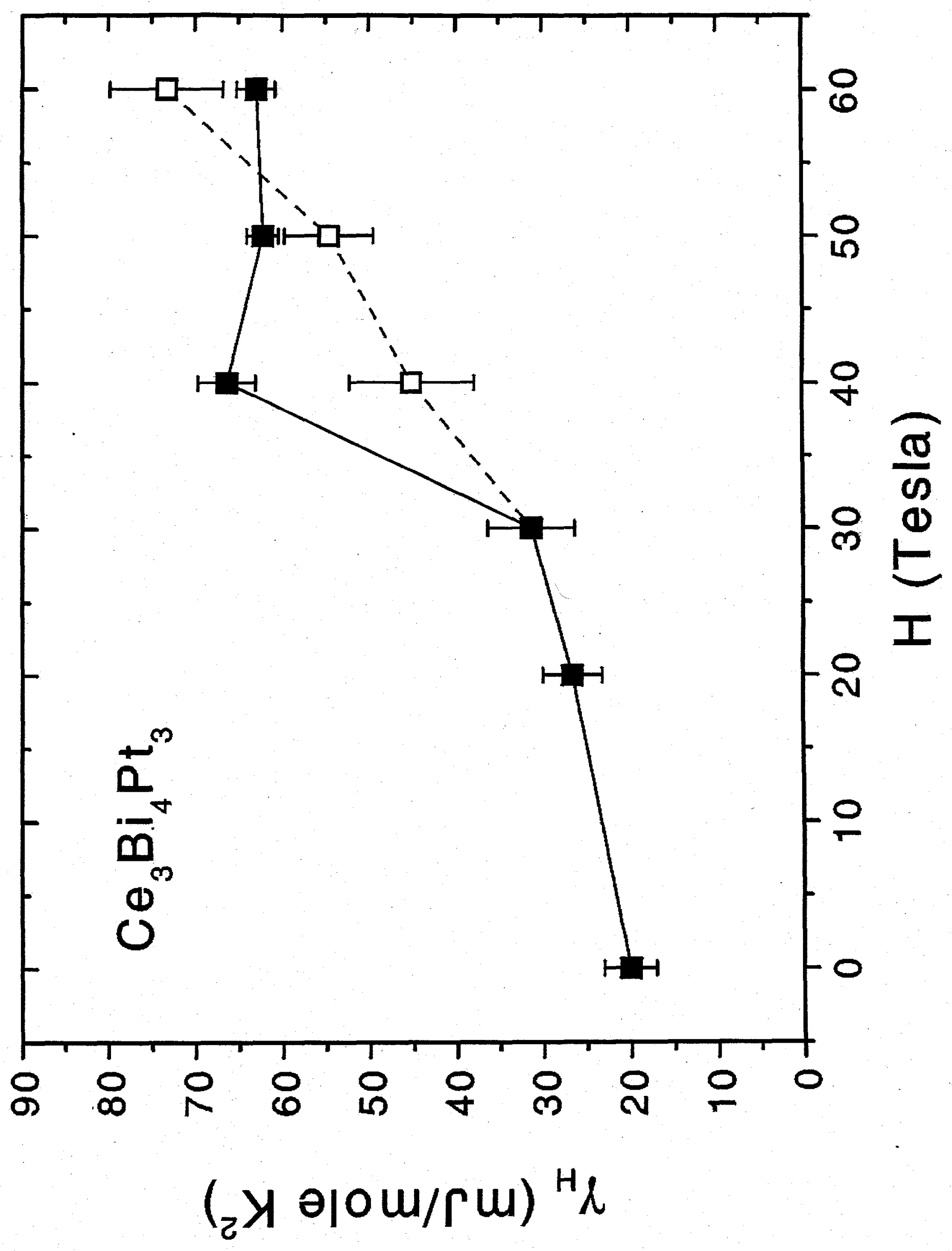


Figure 7

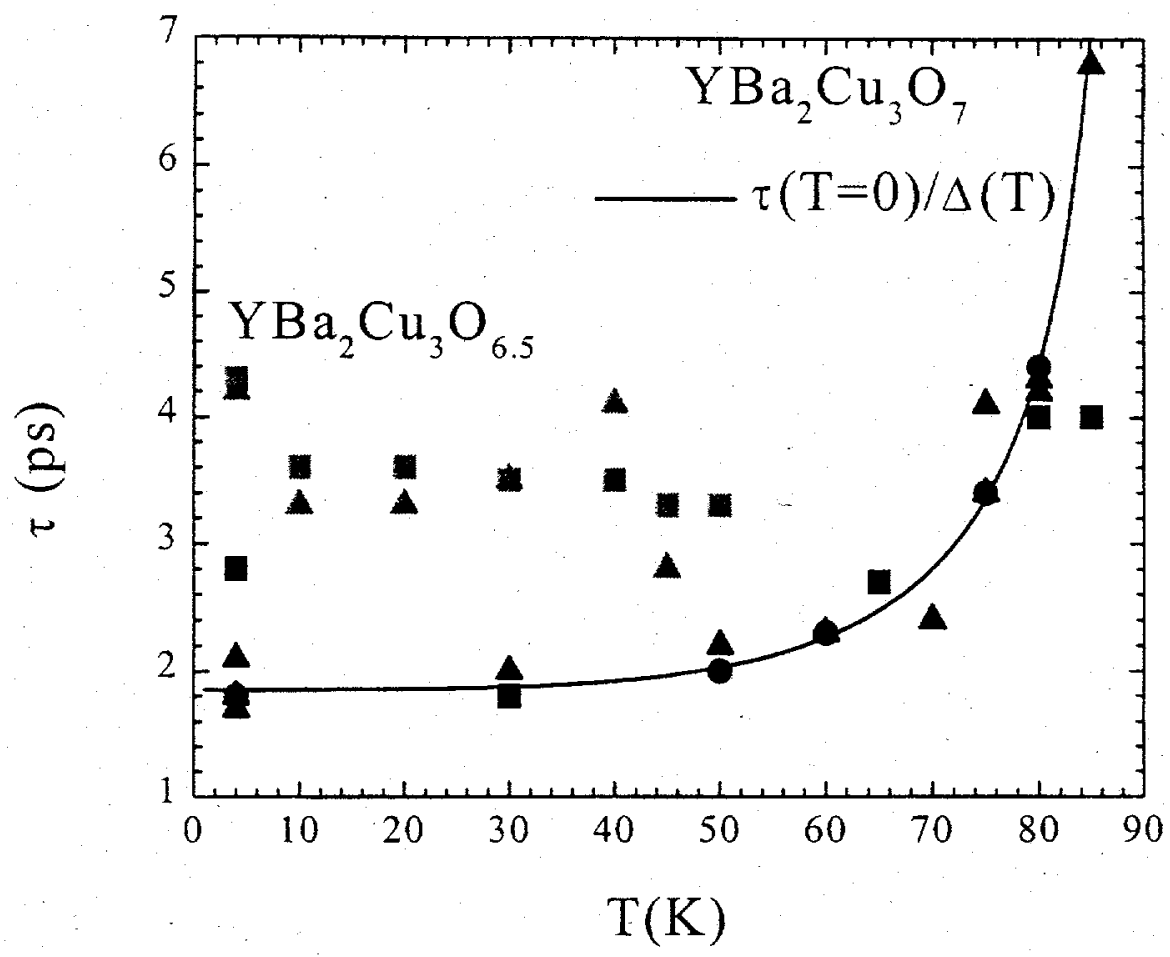


Figure 8

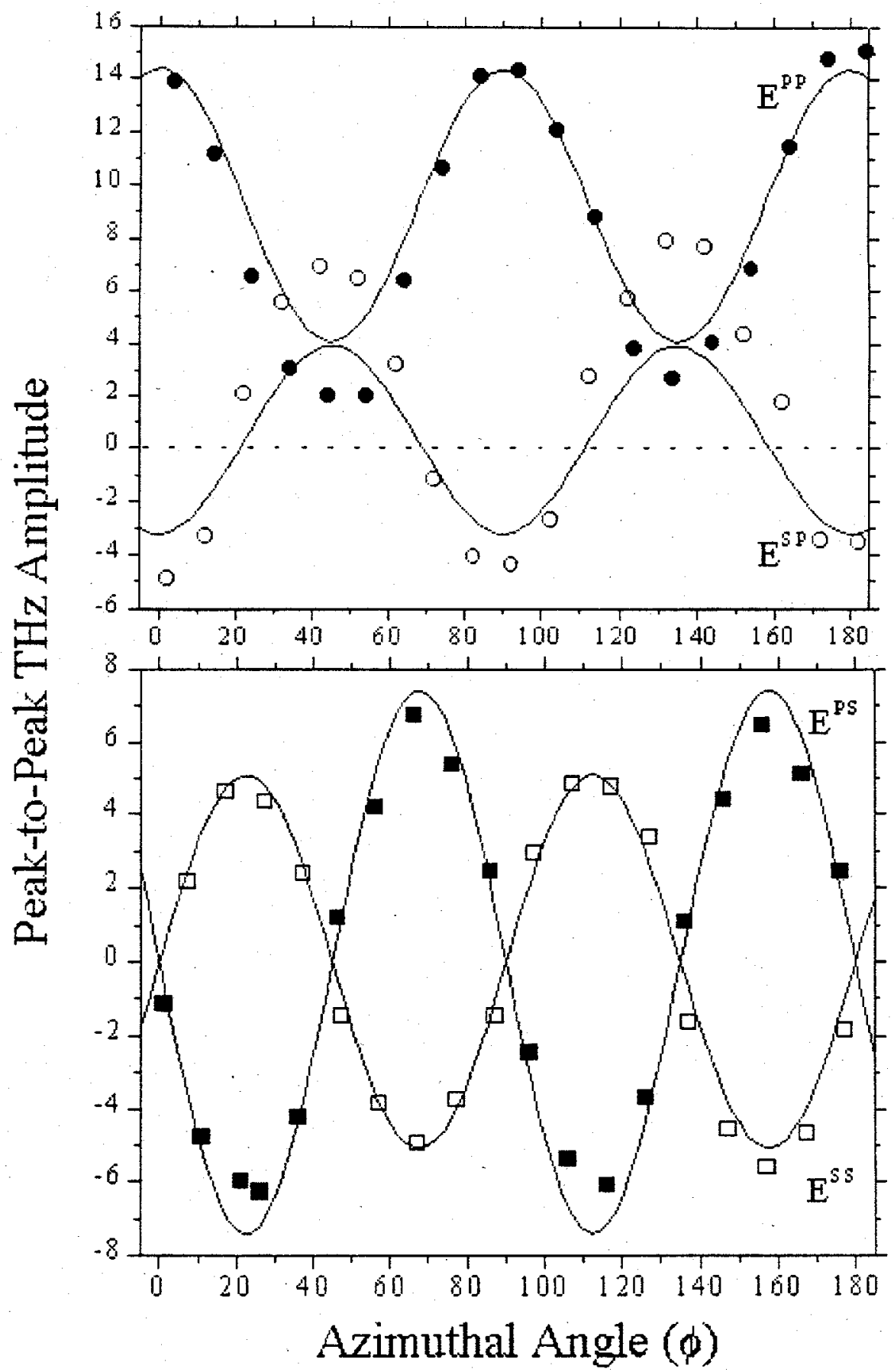


Figure 9

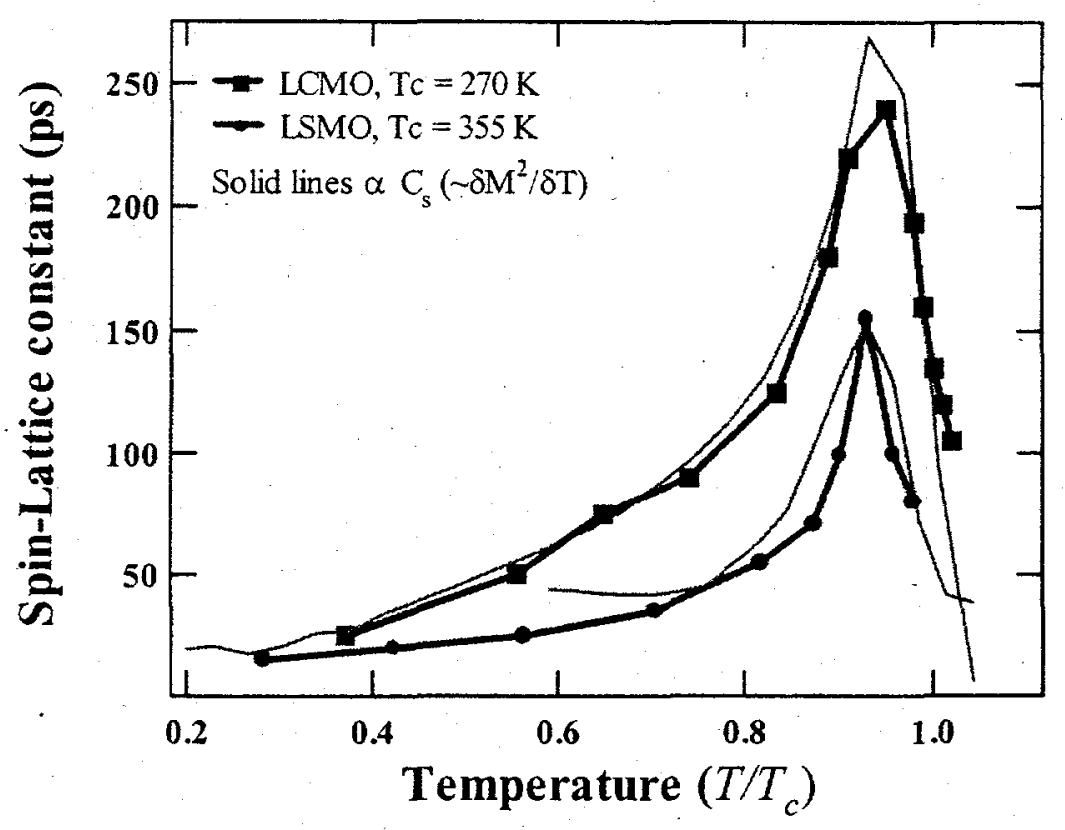




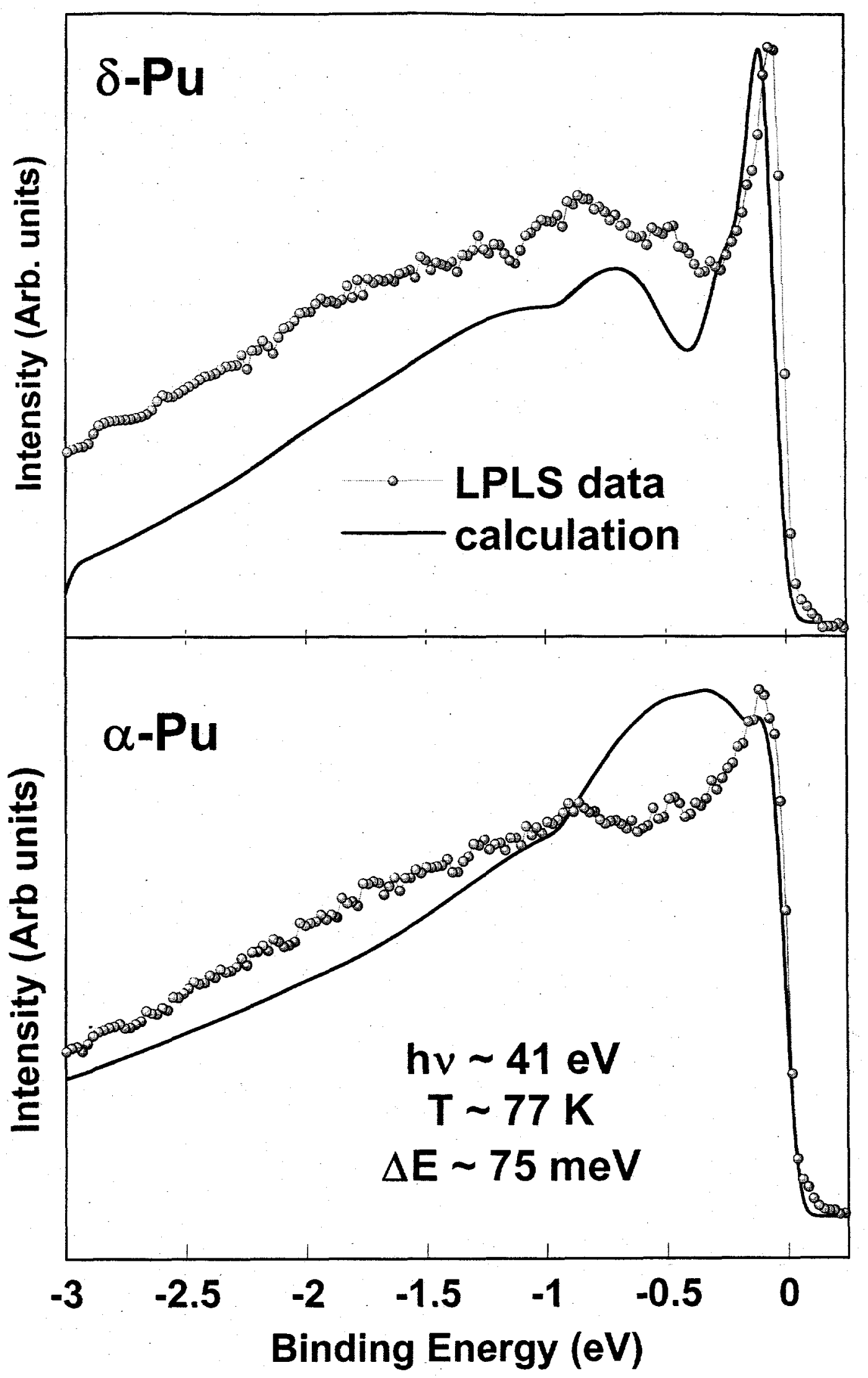




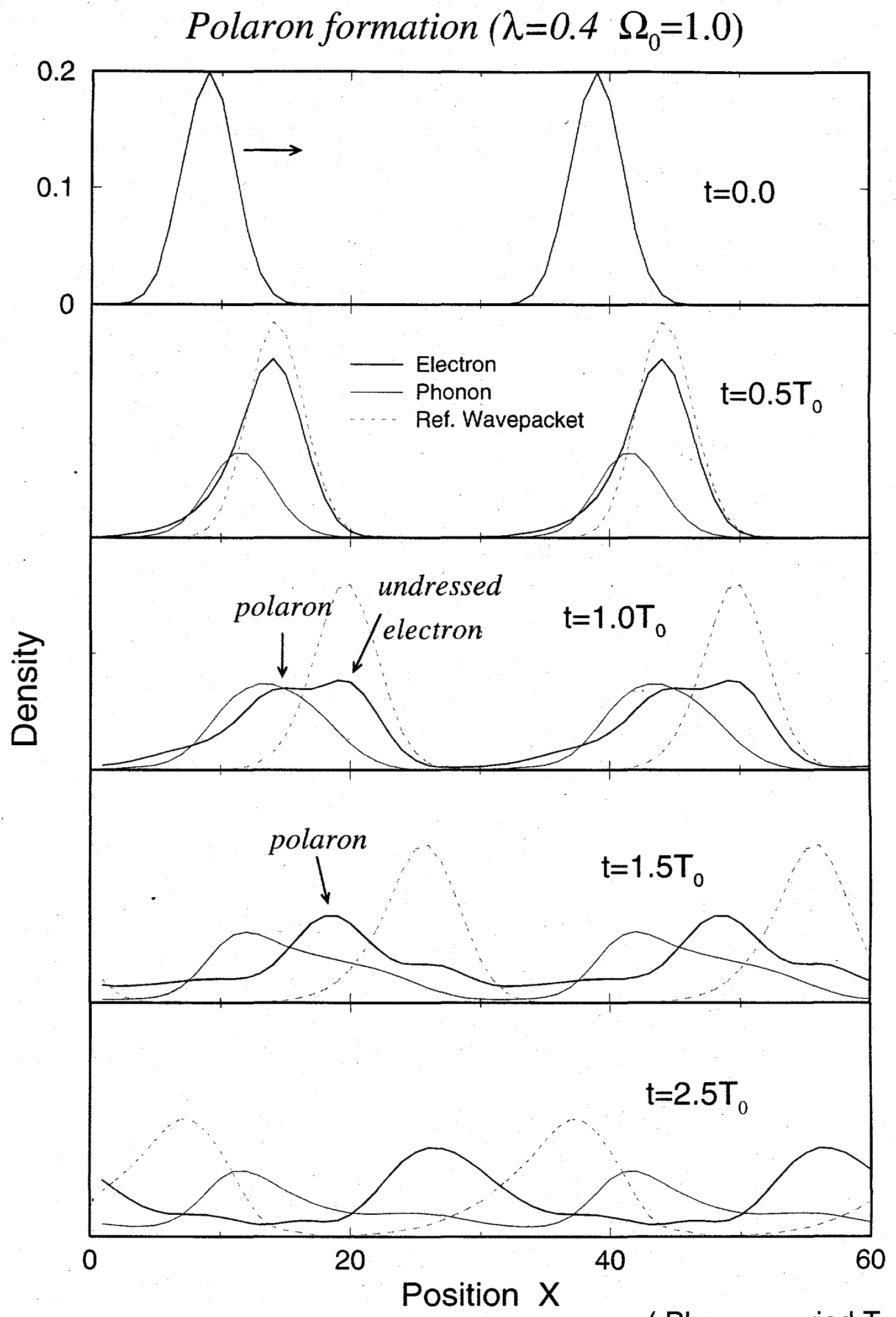

( Phonon period $\mathrm{T}_{0}$ ) 